\title{
Clinical and Imaging Features of Novel Coronavirus Pneumonia in the Elderly Compared with Young and Middle-Aged Individuals
}

\author{
Jia Cao ${ }^{1}$, Jun Zhou ${ }^{1}$, Qingyun Long ${ }^{1,}$, , Gonghao Ling ${ }^{1}$ and Xingnan Liao ${ }^{1}$ \\ ${ }^{1}$ Department of Radiology, Zhongnan Hospital of Wuhan University, Wuhan, China \\ "Corresponding author: Department of Radiology, Zhongnan Hospital of Wuhan University, No.169 Donghu Road, Wuhan, China. Email: longqy2005@126.com
}

Received 2020 April 06; Revised 2020 September 29; Accepted 2020 October 11.

\begin{abstract}
Background: A cluster of patients with coronavirus disease 2019 (COVID-19) pneumonia caused by infection with severe acute respiratory syndrome coronavirus 2 (SARS-CoV-2) were reported worldwide, but there are few reports on age-related computed tomography (CT) signs and clinical characteristics of COVID-19 patients.

Objectives: We aimed to analyze the CT imaging characteristics and clinical features of elderly patients with novel COVID-19 and compare them with young COVID-19 patients, in order to provide imaging basis for clinical diagnosis.

Patients and Methods: The clinical and chest CT imaging data of elderly patients ( $\geq 60$ years) and young patients (age of 20 60 years) with positive novel coronavirus nucleic acid admitted to our hospital from January 28, 2020 to February 16, 2020 were retrospectively analyzed, and the differences in clinical and imaging characteristics between the two groups were compared.

Results: One hundred eighty three COVID-19 patients (96 elderly: mean age, $72.76 \pm 5.65$; 87 young: mean age, $42.69 \pm 12.44$ ) with positive novel coronavirus nucleic acid were retrospectively enrolled. The clinical symptoms of fever (79\%), cough (64\%) and weakness (59\%) in elderly patients were higher than those of young patients $(\mathrm{P}<0.01)$. Asymptomatic young patients $(15 \%)$ were more common than the elderly $(2 \%)(\mathrm{P}<0.01)$. Twenty-four patients $(13 \%)$ had lesions that involved both the periphery and the center of the lung, while the lesions were located in the periphery of the lung in 159 patients (87\%). Ground-glass opacification in young patients $(71 \%)$ was significantly higher than that of the elderly $(26 \%)(\mathrm{P}<0.001)$. Compared with the young group (37\%), the proportion of extensive involvement of lung lobes was higher in the elderly group $(72 \%)(\mathrm{P}<0.001)$.

Conclusion: Elderly and young patients with corona virus disease had some common CT and clinical features, but elderly patients may have relatively severe CT findings, and these differences may be related to the severity and prognosis of COVID-19 pneumonia.
\end{abstract}

Keywords: COVID-19, Imaging Features, Computer Tomography

\section{Background}

The first unexplained case of pneumonia in Wuhan, Hubei province since December 2019 spread rapidly to other provinces and cities in China and other countries in the following weeks. After deep sequencing analysis, the pathogen was found to be a novel coronavirus, International Committee on Taxonomy of Viruses (ICTV) named the novel coronavirus severe acute respiratory syndrome coronavirus 2 (SARS-CoV-2). Meanwhile, the world health organization (WHO) has named the pneumonia infected by the novel coronavirus disease 2019 (COVID-19) (1, 2).

COVID-19 is a respiratory infectious disease, it is mainly transmitted by droplets and contact, and may be transmitted by aerosols after prolonged exposure to high concentrations of aerosols in a relatively closed environment (3). It often causes infectious lesions of different degrees in the lungs. Elderly people and people with chronic underlying diseases are more likely to develop severe pneumonia, which is not conducive to the treatment and rehabilitation of patients $(4,5)$. However, young patients often have mild or asymptomatic clinical symptoms and a better prognosis.

\section{Objectives}

This study retrospectively analyzed the clinical data and imaging manifestations of elderly COVID-19 patients and young COVID-19 patients diagnosed in our hospital, and compared the differences of clinical and imaging characteristics between the two groups. The aim was to determine and analyze the common and different details of the disease in younger patients and older adults, provide imag- 
ing basis for timely diagnosis and early clinical treatment, and avoid the development of refractory severe patients as far as possible.

\section{Patients and Methods}

\subsection{Patient Data}

One hundred eighty three COVID-19 patients with positive novel coronavirus nucleic acid admitted to our hospital from January 28, 2020 to February 16, 2020 were selected, all of which met the relevant diagnostic criteria (5). Exclusion criteria were as follows: (1) Previous history of chronic obstructive pulmonary diseases; (2) pneumonia caused by other pathogenic microorganisms, such as tuberculosis. The clinical and laboratory data were reviewed from the electronic medical records. The chief complaints, symptoms of the disease, and the time interval (in days) from disease onset to computed tomography (CT) examination were recorded. Chest CT images were collected and evaluated using the picture archiving and communication systems (PACS).

\subsection{Chest Thin-Section CT Scan}

In these groups of cases, all patients received thinsection CT scan of the chest. Patients were supine and scanned at the end of inspiration, ranging from the tip of the lung to the bottom of the lung. Scanning was performed using one of the following three instruments: GE discovery, Philips Ingenuity and Siemens Somatom Sensation, with the following parameters: thickness of the layer was $1.25 \mathrm{~mm}$, tube voltage was $120 \mathrm{kV}$, and tube current was $100 \mathrm{~mA}$.

\subsection{Image Interpretation}

The CT images were analyzed separately by two senior radiologists with 5 to 10 years of experience in chest diagnosis, they independently and freely assessed the CT features using both axial CT images and multiplanar reconstruction images, and the final CT features were agreed upon. The following characteristics were evaluated: (1) Number of lobes involved: The minimum involved scope was single lung lobe involved and the maximum involved scope was five lobes involved; (2) lesion distribution: Peripheral areas, and peripheral and central areas (division by $3 \mathrm{~cm}$ under pleura); (3) lesion density (presence of pure ground glass opacity, ground glass opacity and consolidation, crazy paving pattern and the above three types of hybrid); (4) interstitial changes; (5) accompanying signs; (6) pleural effusion.

\subsection{Statistical Analysis}

SPSS version 22.0 (SPSS Inc., Chicago, Il., USA) was used for statistical analysis of the data. Patients were divided into two groups according to age as follows: (1) age younger than 60 years group; (2) age 60 years and older group. Normal distribution test was conducted on the measurement data, normally distributed data were represented by mean \pm standard deviation, then $t$-test was used for comparison. The counting data was expressed by frequencies and percentage, and Fisher's exact probability test was used for comparison (for categorical data). P < 0.05 was considered statistically significant.

\section{Results}

In total, 183 COVID-19 patients (96 elderly: mean age $72.76 \pm 5.65$; 87 young: mean age $42.69 \pm 12.44)$ were identified. One hundred four (56.8\%) were men and 79 (43.2\%) were women, with slightly more men than women. The clinical characteristics, imaging manifestations and opacification distribution of COVID-19 patients are summarized in Tables 1-3. There was no gender difference between the two groups $(\mathrm{P}=0.666)$. Elderly patients often had hypertension (44 patients [46\%]), diabetes (36 patients [38\%]) and other underlying diseases. The most common clinical symptoms were fever (66\%), cough (52\%) and weakness (45\%). There were more young asymptomatic patients (15\%) than elderly patients (2\%). Significant differences in some clinical parameters were found $(\mathrm{P}<0.05)$. In 142 patients (78\%), lymphocyte count decreased, neutrophils increased in 55 patients (30\%), neutrophils decreased in 15 patients (8\%), leucocyte count increased in 31 patients (17\%), leucocyte count decreased in 40 patients (22\%), and C-reactive protein increased in 151 patients (83\%).

Chest CT lesions of some COVID-19 patients are shown in Figures 1-2. Compared with the young group (37\%), the proportion of extensive involvement of lung lobes was higher in the elderly group (72\%). Twenty four patients (13\%) had lesions that involved both the periphery and the center of the lung, while the lesions were located in the periphery of the lung in 159 patients (87\%). The main imaging findings of the young patients were ground-glass opacification (71\%) in the lung, while the elderly patients mainly showed coexistence of ground-glass opacification and consolidation (47\%) and crazy paving pattern (22\%). The elderly patients with severe pneumonia were more common (16\%) than young patients (6\%). In the patients with severe pneumonia, a large amount of exudation occurred in the lungs, and chest CT showed a diffuse distribution 


\begin{tabular}{|c|c|c|c|c|}
\hline Characteristics & Total patients $(n=183)$ & Elderly patients $(\mathrm{n}=96)$ & Young patients $(n=87)$ & Pvalue $^{\mathrm{b}}$ \\
\hline Male: Female & 104:79 & $56: 40$ & $48: 39$ & 0.666 \\
\hline \multicolumn{5}{|l|}{ Symptoms } \\
\hline Fever & $120(66)$ & $76(79)$ & $44(51)$ & $<0.001$ \\
\hline Dyspnea & $63(34)$ & $50(52)$ & $13(15)$ & $<0.001$ \\
\hline Cough & $95(52)$ & $61(64)$ & $34(39)$ & 0.001 \\
\hline Sputum & $75(41)$ & $52(54)$ & $23(26)$ & $<0.001$ \\
\hline Pectoralgia & $72(39)$ & $46(48)$ & $26(30)$ & 0.015 \\
\hline Weakness & $82(45)$ & $57(59)$ & $25(29)$ & $<0.001$ \\
\hline Diarrhea & $17(9)$ & $11(11)$ & $6(7)$ & 0.319 \\
\hline Headache & $28(15)$ & $13(14)$ & $15(17)$ & 0.541 \\
\hline Vomiting & $16(9)$ & $9(9)$ & $7(8)$ & 0.799 \\
\hline No symptom & $15(8)$ & $2(2)$ & $13(15)$ & 0.002 \\
\hline \multicolumn{5}{|c|}{ Time interval (from disease onset to CT examination), d } \\
\hline$\leq 3$ & $78(43)$ & $42(44)$ & $36(41)$ & - \\
\hline$>3, \leq 7$ & $60(33)$ & $32(33)$ & $28(32)$ & - \\
\hline$>7$ & $45(25)$ & $22(23)$ & $23(26)$ & - \\
\hline
\end{tabular}

Abbreviation: COVID-19; coronavirus disease 2019

${ }^{\mathrm{a}}$ Values are expressed as No. (\%).

${ }^{\mathrm{b}}$ Difference among elderly patients and young patients.

\begin{tabular}{|c|c|c|c|c|}
\hline Distribution & Total patients $(n=183)$ & Elderly patients $(n=96)$ & Young patients $(n=87)$ & Pvalue $^{\mathrm{b}}$ \\
\hline Single lobe & $23(13)$ & $5(5)$ & $18(21)$ & 0.002 \\
\hline 2 lobes & $14(8)$ & $6(6)$ & $8(9)$ & 0.58 \\
\hline 3 lobes & $18(10)$ & $8(8)$ & $10(11)$ & 0.62 \\
\hline 5 lobes & $109(60)$ & $72(75)$ & $37(43)$ & $<0.001$ \\
\hline Periphery & $159(87)$ & $92(96)$ & $67(77)$ & $<0.001$ \\
\hline Periphery and center & $24(13)$ & $6(6)$ & $18(21)$ & 0.004 \\
\hline
\end{tabular}

Abbreviation: COVID-19; coronavirus disease 2019

${ }^{a}$ Values are expressed as No. (\%).

${ }^{\mathrm{b}}$ The mean difference is significant at a level of 0.05 .

of large, opacification or interstitial changes in bilateral lungs, sometimes accompanied by atelectasis, pleural effusion, air bronchogenic or tree-in-bud signs. The main characteristic was the diffuse distribution of lesions. Other CT findings included air bronchogram (38\%), thickening of the adjacent pleura (31\%), and lymphadenopathies (9\%).

\section{Discussion}

The novel coronavirus pneumonia is an acute infectious disease caused by infection with SARS-CoV-2. The disease presents obvious population aggregation, mainly family aggregation. Current epidemiological investigations show that the incubation period is generally 3 - 7 days, rarely more than 14 days. The elderly and those with underlying diseases are more seriously ill after infection, leading to severe pneumonia and even death. Adults can be diagnosed with severe pneumonia if they meet any of the following criteria: (1) shortness of breath ( $R R \geq 30 / \mathrm{min}$ ); (2) at rest, oxygen saturation $\leq 93 \%$; (3) arterial partial oxygen pressure $\left(\mathrm{PaO}_{2}\right)$ /oxygen absorption concentration $\left(\mathrm{FiO}_{2}\right) \leq 300 \mathrm{mmHg}(1 \mathrm{mmHg}=0.133 \mathrm{kpa}) ;(4)$ pulmonary 
Table 3. Comparison of Imaging Manifestations of COVID-19 Patients ${ }^{\mathrm{a}}$

\begin{tabular}{|c|c|c|c|c|}
\hline Imaging manifestations & Total patients $(n=183)$ & Elderly patients $(n=96)$ & Young patients $(n=87)$ & Pvalue $^{\text {b }}$ \\
\hline Pure ground-glass opacification & $87(48)$ & $25(26)$ & $62(71)$ & $<0.001$ \\
\hline Ground glass opacification and consolidation & $66(36)$ & $45(47)$ & $21(24)$ & 0.002 \\
\hline Crazy paving pattern & $24(13)$ & $21(22)$ & $3(3)$ & $<0.001$ \\
\hline The above three hybrid types & $6(3)$ & $5(5)$ & $1(1)$ & 0.214 \\
\hline Air bronchogram & $70(38)$ & $38(40)$ & $32(37)$ & 0.761 \\
\hline Bronchiolectasis & $13(7)$ & $13(14)$ & $0(0)$ & $<0.001$ \\
\hline Pleural effusion & $8(4)$ & $6(6)$ & $2(2)$ & 0.283 \\
\hline Lymphadenopathies & $16(9)$ & $10(10)$ & $6(7)$ & 0.443 \\
\hline Thickening of the adjacent pleura & $56(31)$ & $31(32)$ & $25(29)$ & 0.633 \\
\hline Atelectasis & $10(5)$ & $9(9)$ & $1(1)$ & 0.020 \\
\hline
\end{tabular}

Abbreviation: COVID-19; coronavirus disease 2019

${ }^{a}$ Values are expressed as No. (\%).

${ }^{\mathrm{b}}$ The mean difference is significant at a level of 0.05 .
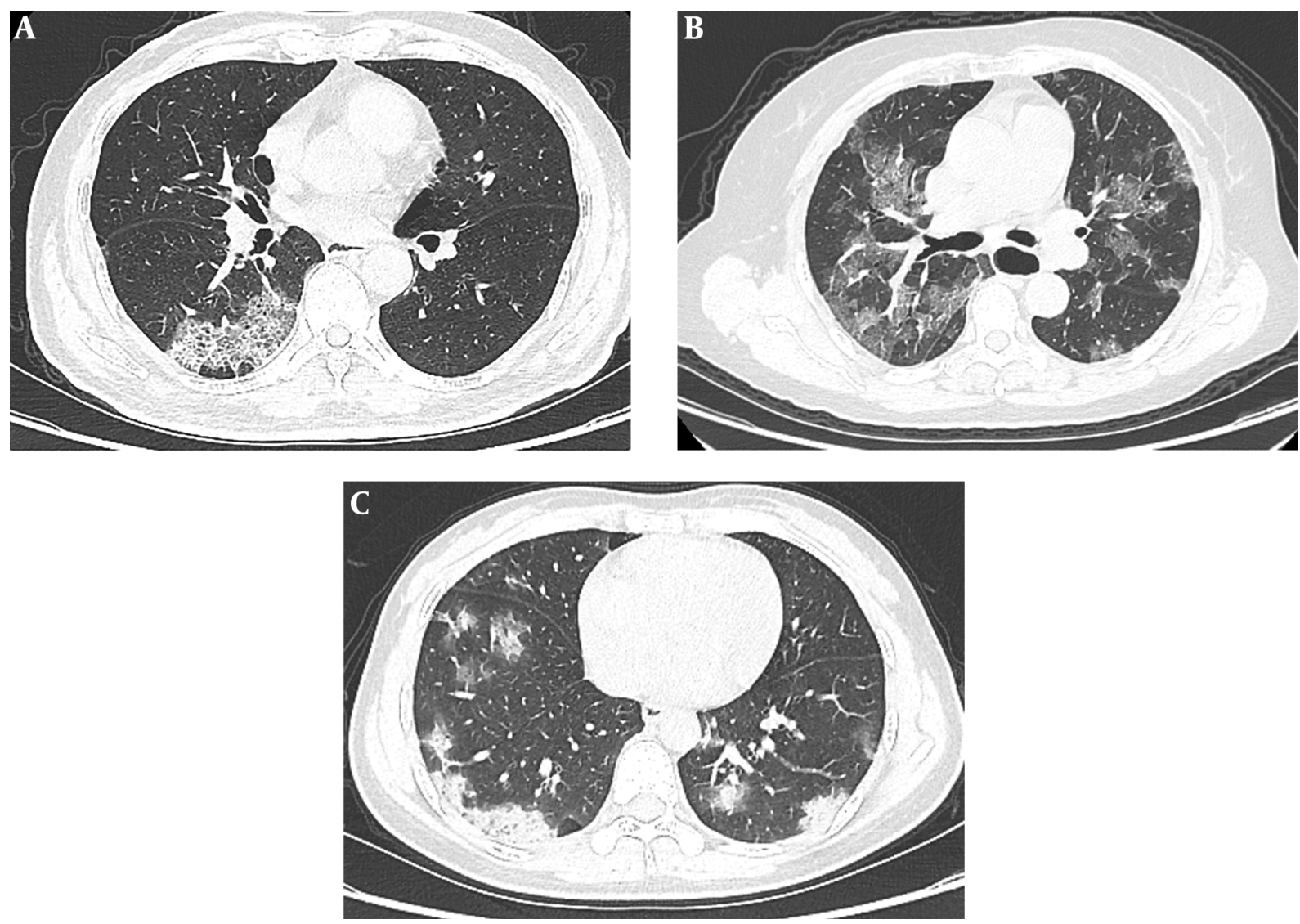

Figure 1. A, A 46-year-old man who presented with fever for 4 days. Axial CT shows interlobular septal thickening in regions of ground glass opacifications (crazy paving pattern); B, A 62-year-old woman who presented with fever and cough for 7 days. CT scan shows multiple ground glass opacification in both lungs; C, A 68-year-old man who presented with fever for 6 days. CT showed patchy ground glass opacification and consolidation in bilateral multiple lobular pattern. 

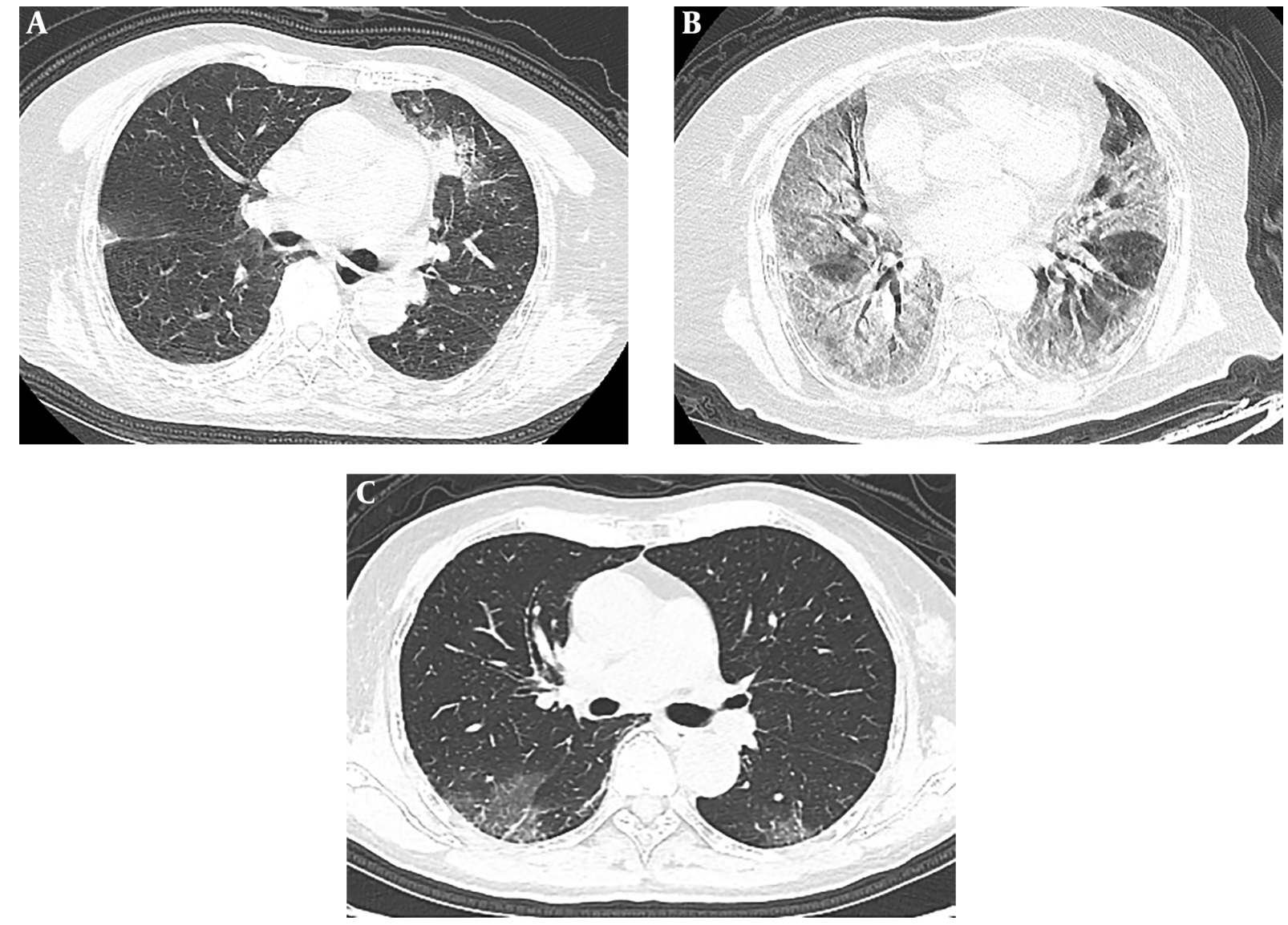

Figure 2. A, A 57-year-old man who presented with fever for 5 days. Axial CT shows ground-glass opacities with consolidation in the left upper lobe; B, A 78-year-old woman who presented with fever and cough for 12 days. She had bilateral white lung, and air bronchogram is detected in the lesion; C, A36-year-old asymptomatic man who has peripheral patchy ground glass opacification in both lungs on CT.

imaging shows that the lesion progresses more than 50\% within 24 to 48 hours (5). Young people tend to have milder symptoms, and children and infants can also develop the disease. Recent reports $(6,7)$ have shown that, the disease tends to occur in men, and the clinical manifestations are mainly fever and dry cough, and some patients may have diarrhea and vomiting. In our study, there were slightly more male patients, and the main symptoms of most COVID-19 patients were fever, cough, and sputum, which is more common in elderly patients.

In this study, there was no significant difference in the distribution of time intervals from disease onset to CT examination between elderly patients and young patients. The interference of different imaging manifestations caused by the course of disease was excluded. Elderly COVID-19 patients are associated with a variety of basic diseases, such as hypertension, and diabetes. Their clinical symptoms are relatively more complex than those of young people. Some studies have shown that the serum antibody concentration of the elderly is reduced and the antibody response to the disease is lower than that of the young. Immune decline and immune aging make elderly COVID-19 patients more serious after infection. Disease progression is faster than young people and easy to develop into severe or critical patients, accounting for a high proportion of patients with severe pneumonia and leading to an increase in case fatality. In terms of imaging findings, chest CT of elderly COVID-19 patients was characterized by mixed ground-glass opacification and consolidation, and their lesions were always in the dynamic change and severe pneumonia in the majority, while the majority of lesions were ground-glass opacification in the group of young patients, and pulmonary consolidation is more common in elderly patients. This may indicate that elderly patients show a more severe inflammatory response and deterioration of lung structure. 
Thin-section CT scan of the chest is especially important for early clinical screening, disease evaluation and efficacy follow-up of COVID-19 patients, and has become a required test item for clinical diagnosis $(8,9)$. Chest CT is mainly characterized by ground-glass opacities and consolidation (10). In the early stage, it is mainly manifested as the patchy ground-glass opacities in the lung, which is mostly distributed in the outer lung zone and under the pleura, and is common in the middle and lower lung lobes. In the progressive stage, multiple ground-glass opacities and consolidation can be seen in both lungs, which can cause grid-like changes and show crazy paving pattern. In some lesions, vascular shadow crossing and air bronchogram can be seen. In the critical stage, the lesions were diffuse (presence of extensive involvement of bilateral lung lobes, ground-glass opacity and ground-glass opacity with consolidation) and even presented as white lung, with bilateral pleural thickening. Pleural effusion and mediastinal lymphadenopathies are rare (11). The imaging findings of COVID-19 are similar to those of severe acute respiratory syndrome (SARS) and Middle East respiratory syndrome (MERS) $(12,13)$. These chest CT findings were mostly distributed in the peripheral bilateral lung field and the subpleural area, probably because the virus mainly affects the terminal bronchioles and lung parenchyma around the respiratory bronchioles in the early stage, which is consistent with the current report $(14,15)$. Some patients also had crazy paving pattern, air bronchogram, bronchiolectasis, pleural effusion, lymphadenopathies, thickening of the adjacent pleura and atelectasis, which are slightly more common among the elderly. The main differential diagnosis of COVID-19 includes mycoplasma pneumonia, cytomegalovirus pneumonia, SARS, MERS and other viral pneumonia.

CT images of COVID-19 patients have comparatively characteristic manifestations. Familiarity with these findings can help us to accurately diagnose COVID-19 pneumonia in different age groups. A small percentage of young people have no clinical symptoms and only imaging findings. Hence, chest thin-section CT scan is particularly important in screening asymptomatic patients with infection. There is a certain false negative rate in RT-PCR test. Some patients who are finally diagnosed as COVID-19 have negative nucleic acid tests in pharynx swabs while their CT scans have characteristic pneumonia manifestations (16). CT should be used as the main imaging method for diagnosis on the basis of nucleic acid detection combined with clinical manifestations and epidemiological history. Familiarity with these will facilitate early diagnosis of COVID-
19 and timely isolation of patients, guide treatment and assess efficacy, and contain the spread of the epidemic. Due to the small sample size and short time in this study, CT images of the entire treatment process of COVID-19 patients were not analyzed, which needs to be further studied in future clinical work.

In conclusion, pulmonary coexistence of ground-glass opacities and consolidation are relatively common in the elderly patients. Existence of underlying diseases in these patients tends to increase the possibility of developing into severe pneumonia. However, ground-glass opacification is more common in young patients. The proportion of extensive involvement of lung lobes was higher in the elderly group compared to the young group. It can be seen from the difference that the clinical symptoms and imaging manifestations of the elderly are more serious, indicating that old age, male sex and complications may be important factors affecting the severity and therapeutic effect of COVID-19 patients.

\section{Footnotes}

Authors' Contributions: Jia Cao carried out the study and design, collected important background information, data acquisition, data analysis, and drafted the manuscript. Gonghao Ling and Xingnan Liao provided assistance for literature search, data acquisition, data analysis, and manuscript editing. Qingyun Long and Jun Zhou performed design of the article, data analysis, and manuscript review. All authors have read and approved the final manuscript.

Conflict of Interests: All authors declare that they have no conflict of interest.

Ethical Approval: Ethical approval was given by the local hospital ethical committee (code: 2020-010).

Funding/Support: No funding.

Informed Consent: Informed consent was obtained from the patients and their family.

\section{References}

1. World Health Organization. WHO Director-General's remarks at the media briefing on 2019-nCoV. 2020, [cited 2020 Feb 11]. Available from: https://www.who.int/dg/speeches/detail/who-director-general-sremarks-at-the-media-briefing-on-2019-ncov-on-11-february2020.

2. World Health Organization. Coronavirus disease (COVID-19) outbreak. 2020, [cited 2020 Feb 15]. Available from: https://www.who.int/ emergencies/diseases/novel-coronavirus-2019. 
3. Huang C, Wang Y, Li X, Ren L, Zhao J, Hu Y, et al. Clinical features of patients infected with 2019 novel coronavirus in Wuhan, China. Lancet. 2020;395(10223):497-506. doi:10.1016/s0140-6736(20)30183-5.

4. World Health Organization. Clinica imanagement of severe acute respiratory infection when novel coronavirus( $n \mathrm{CoV})$ infectionis suspected. 2020, [cited 2020 Jan 28]. Available from: https://www.who.int/publications-detail/clinical-managementof-severe-acute-respiratory-infection-when-novel-coronavirus(ncov)-infection-is-suspected(accessed.

5. China National Health Commission. Diagnosis and treatment of pneumonitis caused by new coronavirus (trial version 7). Beijing: China National Health Commission; 2020, [cited 2020 Mar 4]. Available from: http://www.nhc.gov.cn/yzygj/s7653p/202001/ 4294563ed39b31739bd0785e67.shtml.

6. Li Q, Guan X, Wu P, Wang X, Zhou L, Tong Y, et al. Early Transmission Dynamics in Wuhan, China, of Novel Coronavirus-Infected Pneumonia. N Engl J Med. 2020;382(13):1199-207. doi: 10.1056/NEJMoa2001316. [PubMed: 31995857]. [PubMed Central: PMC7121484].

7. Xu X, Yu C, Qu J, Zhang L, Jiang S, Huang D, et al. Imaging and clinical features of patients with 2019 novel coronavirus SARS-CoV-2. Eur J Nucl Med Mol Imaging. 2020;47(5):1275-80. doi: 10.1007/s00259-020-047359. [PubMed: 32107577]. [PubMed Central: PMC7080117].

8. Ai T, Yang Z, Hou H, Zhan C, Chen C, Lv W, et al. Correlation of Chest CT and RT-PCR Testing for Coronavirus Disease 2019 (COVID19) in China: A Report of 1014 Cases. Radiology. 2020;296(2):E32-40. doi: 10.1148/radiol.2020200642. [PubMed: 32101510]. [PubMed Central: PMC7233399].

9. Pan F, Ye T, Sun P, Gui S, Liang B, Li L, et al. Time Course of Lung Changes at Chest CT during Recovery from Coronavirus Disease 2019 (COVID19). Radiology. 2020;295(3):715-21. doi: 10.1148/radiol.2020200370. [PubMed: 32053470]. [PubMed Central: PMC7233367].
10. Chung M, Bernheim A, Mei X, Zhang N, Huang M, Zeng X, et al. CT Imaging Features of 2019 Novel Coronavirus (2019-nCoV). Radiology. 2020;295(1):202-7. doi: 10.1148/radiol.2020200230. [PubMed: 32017661]. [PubMed Central: PMC7194022].

11. Shi H, Han X, Jiang N, Cao Y, Alwalid O, Gu J, et al. Radiological findings from 81 patients with COVID-19 pneumonia in Wuhan, China: a descriptive study. Lancet Infect Dis. 2020;20(4):425-34. doi: 10.1016/s14733099(20)30086-4.

12. Wong KT, Antonio GE, Hui DS, Lee N, Yuen EH, Wu A, et al. Severe acute respiratory syndrome: radiographic appearances and pattern of progression in 138 patients. Radiology. 2003;228(2):401-6. doi: 10.1148/radiol.2282030593. [PubMed:12759474].

13. Das KM, Lee EY, Langer RD, Larsson SG. Middle East Respiratory Syndrome Coronavirus: What Does a Radiologist Need to Know? AJR Am J Roentgenol. 2016;206(6):1193-201. doi: 10.2214/AJR.15.15363. [PubMed: 26998804].

14. Kanne JP. Chest CT Findings in 2019 Novel Coronavirus (2019-nCoV) Infections from Wuhan, China: Key Points for the Radiologist. Radiology. 2020;295(1):16-7. doi: 10.1148/radiol.2020200241. [PubMed: 32017662]. [PubMed Central: PMC7233362].

15. Yoon SH, Lee KH, Kim JY, Lee YK, Ko H, Kim KH, et al. Chest Radiographic and CT Findings of the 2019 Novel Coronavirus Disease (COVID-19): Analysis of Nine Patients Treated in Korea. Korean J Radiol. 2020;21(4):494-500. doi: 10.3348/kjr.2020.0132. [PubMed: 32100485]. [PubMed Central: PMC7082662].

16. Xie X, Zhong Z, Zhao W, Zheng C, Wang F, Liu J. Chest CT for Typical Coronavirus Disease 2019 (COVID-19) Pneumonia: Relationship to Negative RT-PCR Testing. Radiology. 2020;296(2):E41-5. doi: 10.1148/radiol.2020200343. [PubMed: 32049601]. [PubMed Central: PMC7233363]. 